\title{
Alternative loading functions in the MSB pollution flushing model
}

\author{
S. Mecca ${ }^{1}$, R. Barber ${ }^{2} \&$ J. Tombarello ${ }^{1}$ \\ ${ }^{I}$ Department of Engineering-Physics-Systems, Providence College, \\ Providence, USA \\ ${ }^{2}$ Computational Engineering Group, CCLRC Daresbury Laboratory, \\ Warrington, $U K$
}

\begin{abstract}
The MSB model of pollution flushing from a tidal basin, which is based on the analytical tidal prism model developed by Barber, has been applied to embayments with non-constant bathymetry and constant pollution loading. This paper extends the versatility of the MSB model by including alternative pollution loading functions. The model, written in Stella (Stella is a software product and trademark of High Performance Systems, Inc. Hanover, NH 03755 USA), can use both the internal functions of Stella as well as a user-defined functional representation that is part of the Stella toolset. The morphology of a pulse function applied at different times throughout the tidal cycle is studied. Results of these simulations are applied to answer a hypothetical yet useful question: if a certain mass of pollutant must be loaded into a tidal embayment, when should this loading be applied to minimize the time for the concentration to 'relax' to a certain critical level? These results and the multiple possibilities for extending the MSB model are explored.
\end{abstract}

Keywords: tidal prism, pollution flushing, tidal embayment.

\section{Introduction}

Modeling the flushing of pollution from an ideal tidal basin, which is loaded at a constant rate, has been studied both numerically [1] and analytically [2] using a simple formulation relating to the following mass flow equation: 


$$
\frac{d(C V)}{d t}=V \frac{d C}{d t}+C \frac{d V}{d t}=Q C+k
$$

where $Q(t)$ is the discharge through the entrance to the embayment, $C(t)$ is the instantaneous concentration, $V(t)$ is the volume of the embayment, and $k$ is the pollution loading rate. During the ebb flow period, $d V / d t=Q$, canceling the $Q C$ term on the right of eqn. (1) with the $C d V / d t$ term on the left. During the flood flow period, there is no $Q C$ term in the equation. This results in two rate equations for $d C / d t$, one for the ebb and another for the flood conditions. The MSB model [1], which is written in Stella, employs the basic state variable approach used in Systems Dynamics to represent these rate equations treating the time-varying pollutant concentration in the embayment as a level. This model also allows for non-rectangular basin geometry and has been applied to real basins with known bathymetry. The model has been further extended to include cascading basins [3]. In the present study, the MSB model has been formulated to allow for non-constant pollution loading and the resulting model has been used to study the effects of loading a fixed mass of pollution into a basin at different times during the tidal cycle and observing relaxation times for the resulting pollutant concentration in the embayment.

\section{Model embellishments}

The single embayment MSB model referenced above is shown in Stella graphical form in Fig. 1. For simplicity, the ebb and flow concentration rates are written as a single biflow rate, $C_{\text {rate }}$, with the logical condition:

$$
\text { if }\left(V_{\text {rate }}>0\right) \text { then }\left(k-C^{*} V_{\text {rate }}\right) / V \text { else } k / V
$$

where $V_{\text {rate }}$ is $d V / d t$ and is positive on the flood tide and negative on the ebb.

In this paper, the pollution loading rate, $k$, will be treated as a function of time. An interface was developed to facilitate user input and observe the output from the simulations. This interface is shown in Fig. 2. The model was run with simple pollution loading functions including a square pulse-, a triangular rampand an instantaneous pulse, representing hypothetical ways in which a pollutant can be released into an embayment. The pollution concentrations resulting from the release of a fixed mass of pollution 4 hours following the start of the ebb cycle are shown in Fig. 3 for a simple rectangular basin of length, width and mean depth of $432 \mathrm{~m}, 432 \mathrm{~m}$ and $6 \mathrm{~m}$ respectively experiencing a $4 \mathrm{~m}$ tidal range. The pulse function is an 'instantaneous' release delivered over a time equal to the time step used in the simulation, in this case, 0.005 hours.

\section{Analysis of pollution relaxation and release times}

Consider a pollutant released at a constant rate beginning at $t=t_{i}$ and ending at $t=t_{f}$, with a release time, $t_{r}$, being defined as the mid point of $t_{f}-t_{i}$. We first observe the maximum concentrations, $C_{\max }$, as a function of release time. These 


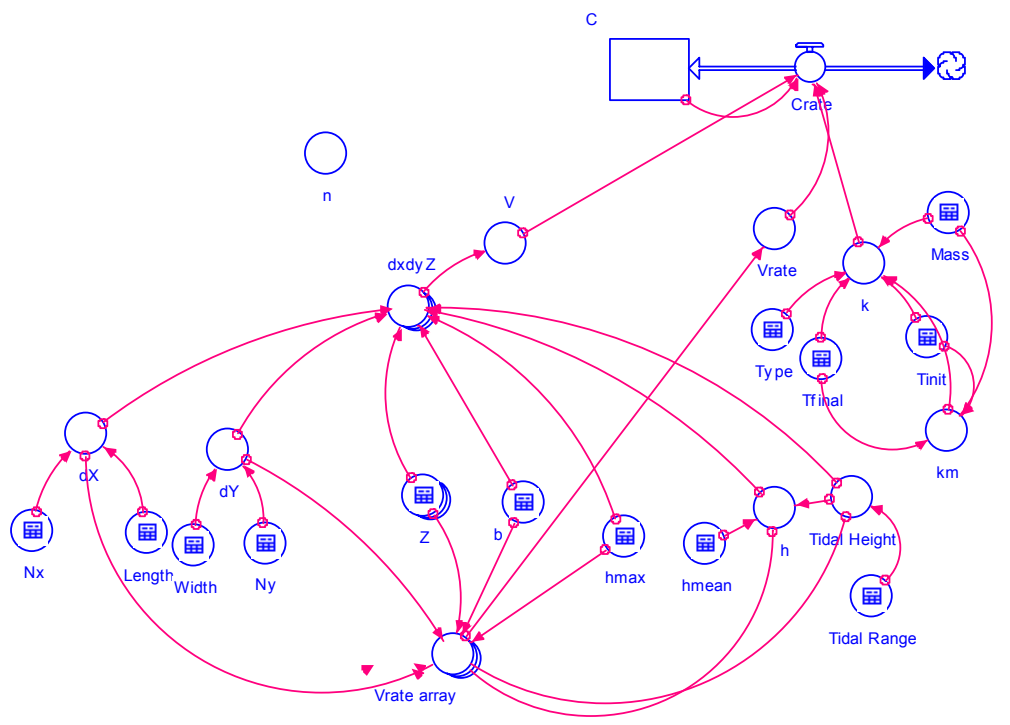

$C(t)=$ Concentration of pollution

CrateFlood $=$ Rate of change in pollution concentration during flood tide .

CrateEbb $=$ Rate of change in pollution concentration during ebb tide.

$b=$ Pollution return flow factor

$\mathrm{k}=$ Pollution loading rate (mass per unit time)

$\mathrm{km}=$ The highest value of $\mathrm{k}$

Length $=$ Length of the grid extending to the limits of the embayment.

Mean_Water_Depth $=$ Average water depth at mean tide.

$\mathrm{n}=$ Internal variable used to keep track of tidal cycles

Period $=12.42$ hours in a tidal cycle

Tidal_Height $=$ Maxtidal swing $(\mathrm{m})$

Tidal_Range $=$ Total tidal swing from low tide and high tide $(\mathrm{m})$

$V=$ Volume of the embayment $(\mathrm{m} 3)$

Vrate $=$ Change in volume $V$ over time based on tides

Width $=$ Width of the grid extending across the embayment

$\mathrm{dX}=$ Length of grid block

$d x d y z[i, j]=$ Volume of every block in the embayment grid at time (t)

$d Y=$ Width of grid block

$\mathrm{h}=$ Average height at time $(\mathrm{t})$

$\mathrm{hmax}=$ The greatest bay depth at high tide at deepest point.

hmean $=$ Average bay depth

$\mathrm{Nx}=$ Number of rows in the embayment grid

$\mathrm{Ny}=$ Number of columns in the embayment grid

Width= Width of the grid extending across the embayment

$Z[i, j]=$ Bathymetric data array for embayment

Mass $=$ The total amount of pollutant to be dumped during a tidal cycle $(\mathrm{kg})$

Tinit=The time at which the polluting starts (hrs)

Tfinal=The time at which the polluting ends (hrs)

Type=This paramter decides the type of loading function to be used.

Type 1 is a constant loading

Type 2 is a user definable step function that is 0 until Tinit and is a constant value between Tinit and Tfinal and 0 again after Tfinal.

Type 3 is an inclining ramp function with a definable start and end time.

Type 4 is an instaneous pulse.

Figure 1: $\quad$ MSB single basin model in Stella. 


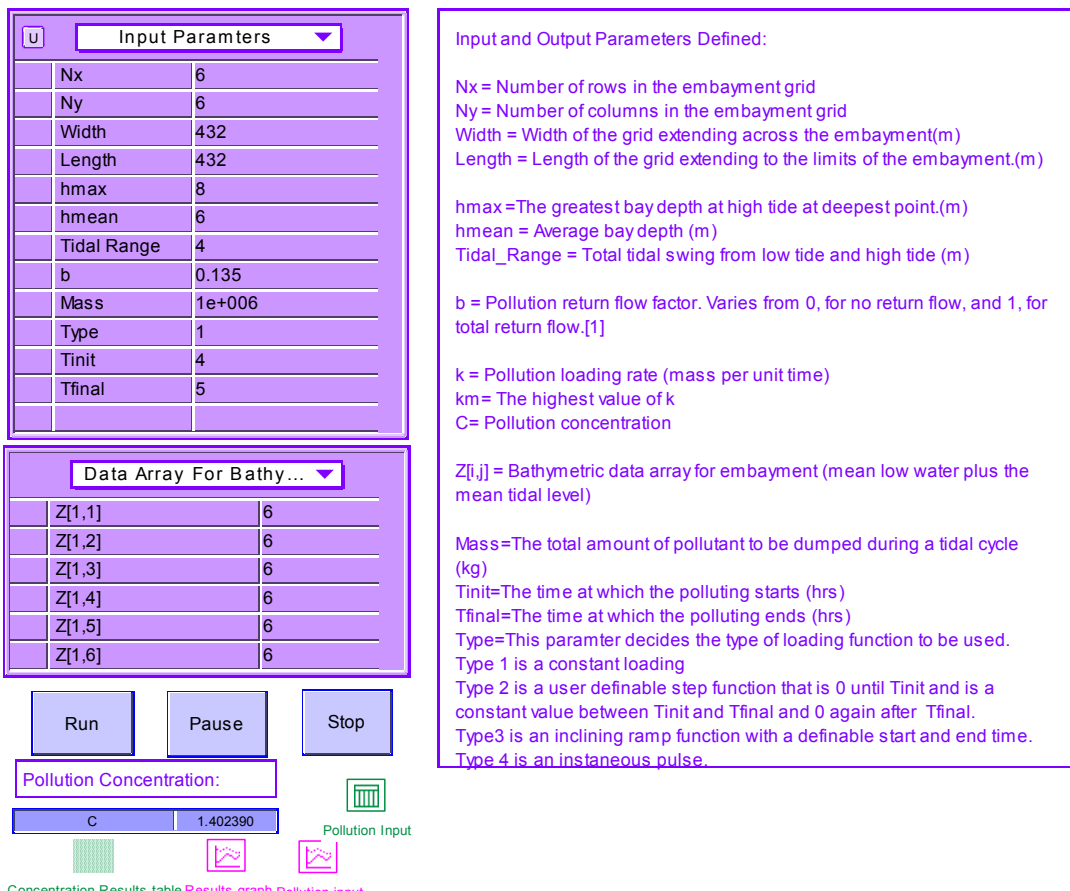

Figure 2: User interface for the single basin MSB model.

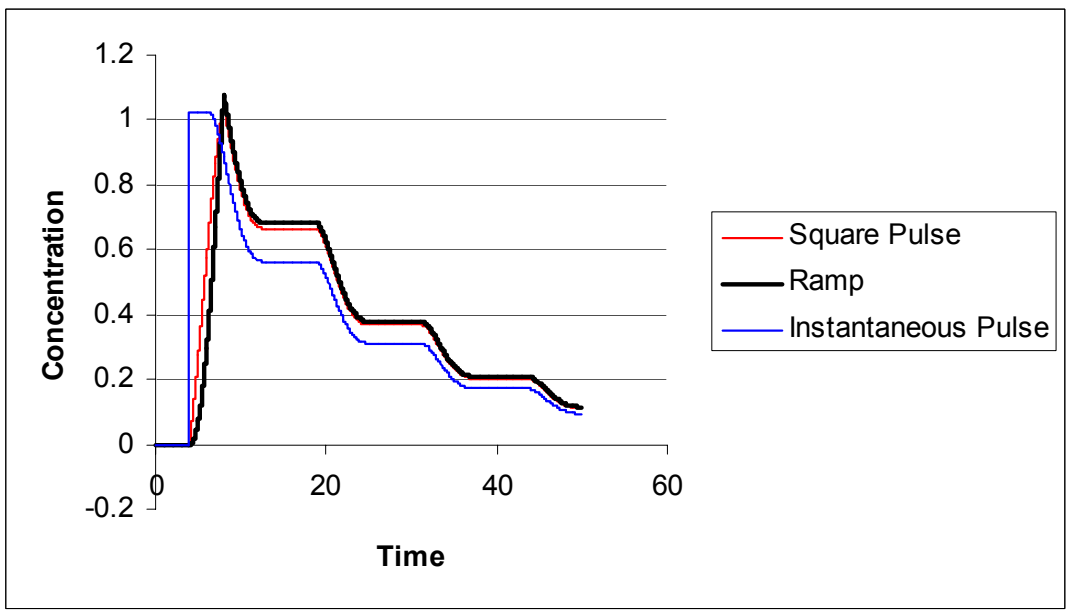

Figure 3: Results of MSB model with equal pollutants released with square pulse, ramp and instantaneous pulse functions at $t=4$ hours following the start of the ebb tide. 
are shown in Fig. 4 for different values of $b$, the pollution return factor that takes into account the amount of discharged pollution returning to the basin on the flood cycle $[2,3]$. In the present analysis, $b$ is used to modify the volume of the tidal prism to account for the return of polluted water during the tidal flood; this methodology has been discussed by Barber [2] and more theoretically by Sanford et al. [4]. The volume modifying factor is taken as (1-b), where $b$ varies between 0 , for no return flow, and 1, for total return flow. Not surprising in Fig. 4 is the symmetry of the curves with $C_{\text {max }}$ reaching a peak when the pollutant is released at the end of the ebb cycle. Somewhat surprising, at first glance is the fact that the magnitudes of the $C_{\max }$ curves are not the same for each value of $b$ since the release times all take place before return pollutant flow is a factor. The different magnitudes result from the aforementioned treatment of return flow, which modifies the volume of the tidal prism. The differences shown in Fig. 4 are the result of these volume modifications. We return to this point and present results of an alternative model formulation, which more closely reflects the physical situation, at the end of this section.

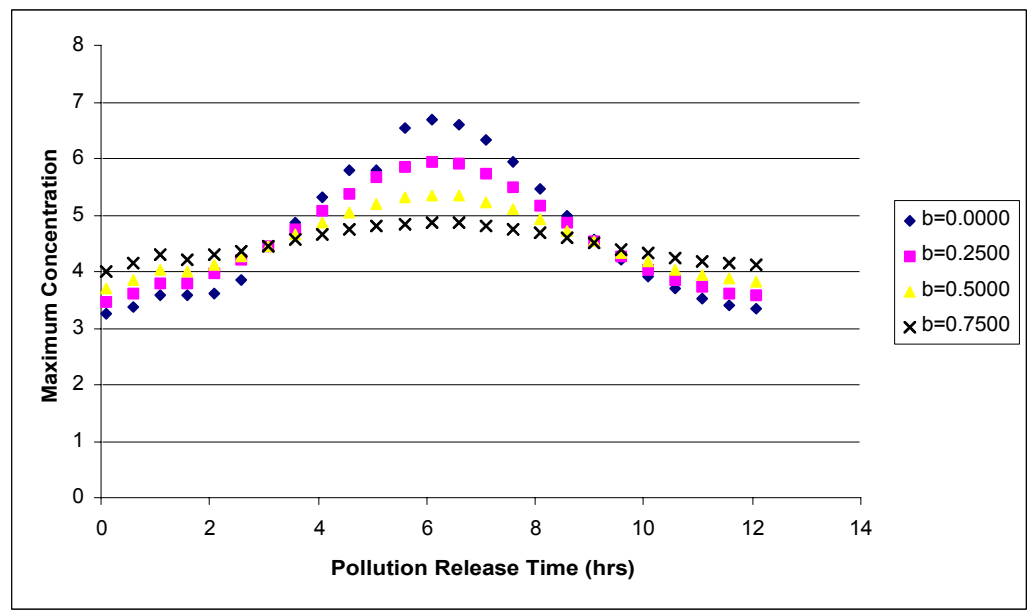

Figure 4: Maximum pollutant concentrations as a function of release time for different values of the pollution return parameter, $b$.

Turning our attention to the degradation of embayment pollution with time, we define a pollution relaxation time, $t_{\text {relax }}$, as the time (measured from the time of initial release) for the concentration to reduce to a certain fraction of the maximum concentration, $C_{\max }$, i.e., to a fraction, $C / C_{\max }$. The model was run parametrically for various values of $b$ and $C / C_{\max }$ and the resulting values of $t_{\text {relax }}$ were extracted. Sample results for $b=0.0$ and $b=0.25$ are shown in Fig. 5 .

Each of the graphs in Fig. 5 gives results spanning a factor of 10 in the relaxation-defining parameter, $C / C_{\max }$. Results are shown for $C / C_{\max }$ fractions of $0.025,0.050,0.100,0.200$ and 0.250 . Attention is drawn to the discontinuity in 
each of the curves with a characteristic minimum relaxation time occurring at pollutant release times prior to the break points depending on the $C / C_{\max }$ fraction and, of course, the value of $b$, the pollution return parameter. An extensive morphological analysis of these minimum relaxation times yields the set of results displayed in Fig. 6, which gives the minimum relaxation time as a function of $b$ for different values of $C / C_{\max }$.

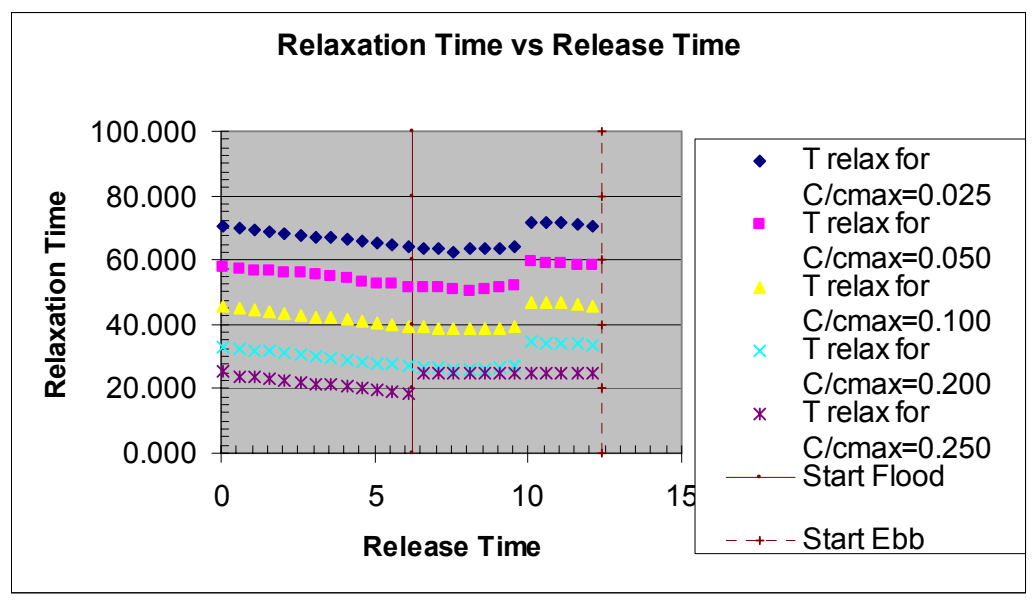

(a) $b=0.0$

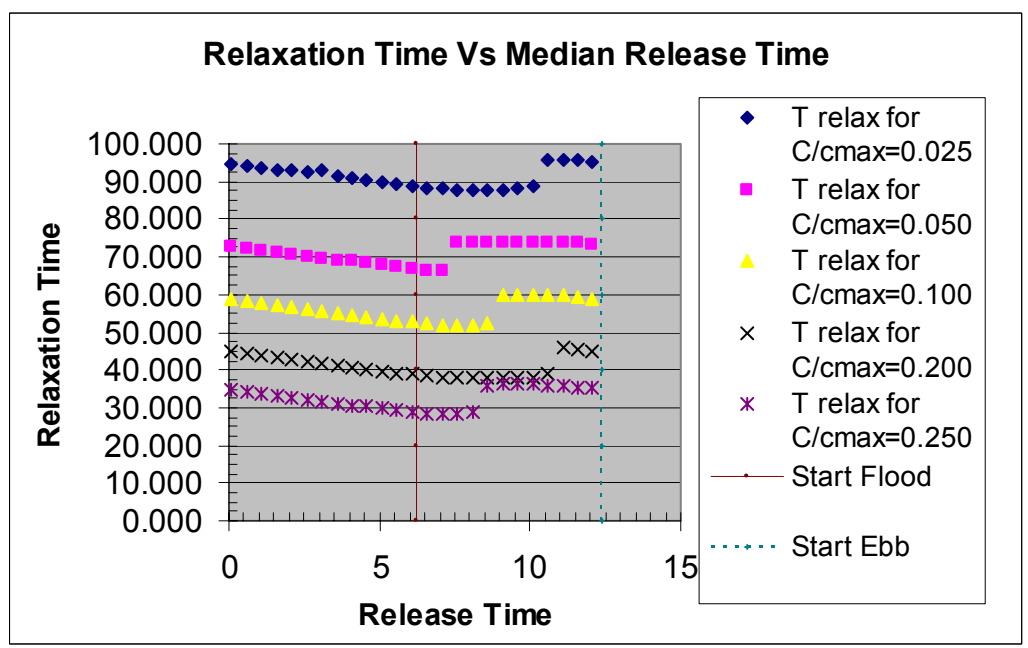

(b) $b=0.25$

Figure 5: Relaxation time as a function of pollutant release time for $b=0.0$ (upper graph) and $b=0.25$ (lower graph). The results are shown for relaxation-defining fractions, $C / C_{\max }$ of $0.025,0.050,0.100,0.200$ and 0.250 from the top series to the bottom series respectively in each graph. 


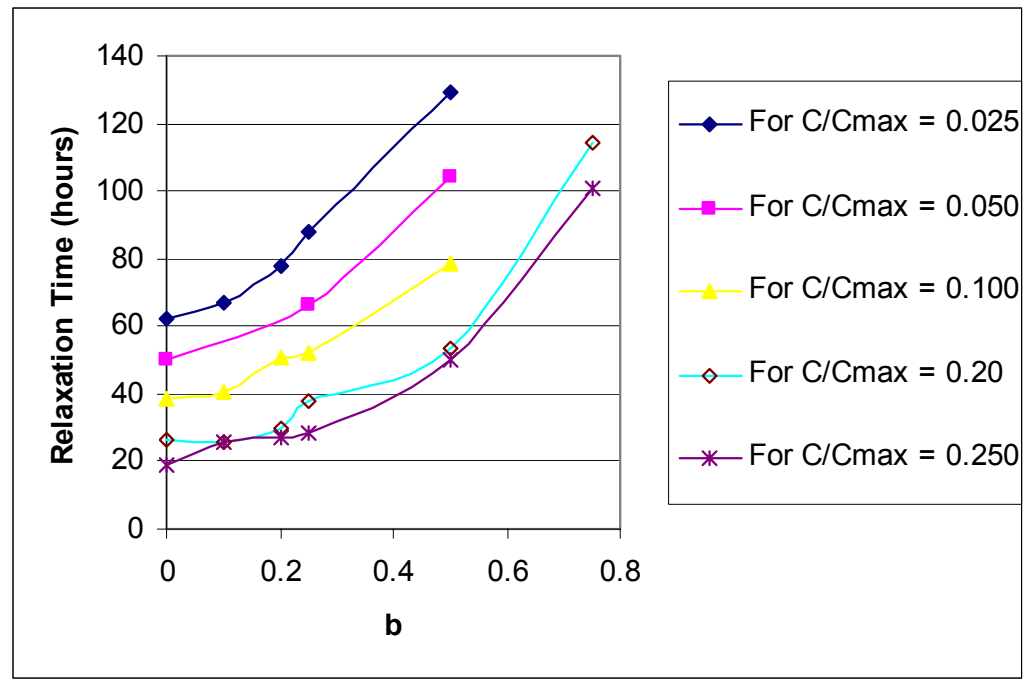

Figure 6: Minimum relation times as a function of $b$ for different relaxationdefining fractions, $C / C_{\max }$.

Inasmuch as relaxation times are strongly influenced by the parameter $b$, it is reasonable to ask how one might determine $b$ for a given embayment. This question has recently been addressed in a study of the MSB model for cascading basins [3] where $b$ is related to the ratio between the volume of the inner pollution-loaded basin and the volume of the subsequent basin, in a sequence of embayments. In the present study, the pollution return factor $b$ is used to modify the volume of the tidal prism to account for the return flow. However, an alternative approach for modeling pollution return phenomena has been described by Barber [2]. This alternative formulation accounts for the return flow by incorporating $b$ into the governing pollution flow equations rather than modifying the volume of the tidal prism. The philosophy behind this methodology is that the formulation aligns more closely to the physical processes involved in real embayments. However, it has been found that the differences between the predicted relaxation times using the two formulations are negligible, even for relatively large values of $b$.

\section{Application to marine operations and environmental problems}

The MSB model and the analysis presented in this study can help answer questions relating to marine operations that have ecological or environmental considerations. For example, if a certain mass of pollutant must be released into a tidal embayment as a result of a marine operation such as aquaculture, mariculture, or an industrial processing operation, when should this pollutant be discharged to minimize the time for the concentration to 'relax' to a certain 
critical level? A specific example would be the release of sewerage from a holding tank that must be readied for continuing critical processing; such a release might threaten shell-fishing in the embayment if the pollutant concentration exceeded a certain level. One might wish to know when the tank should be released in order to minimize the time required for the concentration to reach a level acceptable for the resumption of shell fishing. Using the analysis presented in the previous section, one needs to know the critical 'acceptable' concentration or $C / C_{\max }$, the value of $b$, the pollution return parameter, and the embayment geometry. Then, assuming the basin satisfies the basic conditions of the MSB model, e.g., the basin is well-mixed, simulations can be used to obtain the optimum release time.

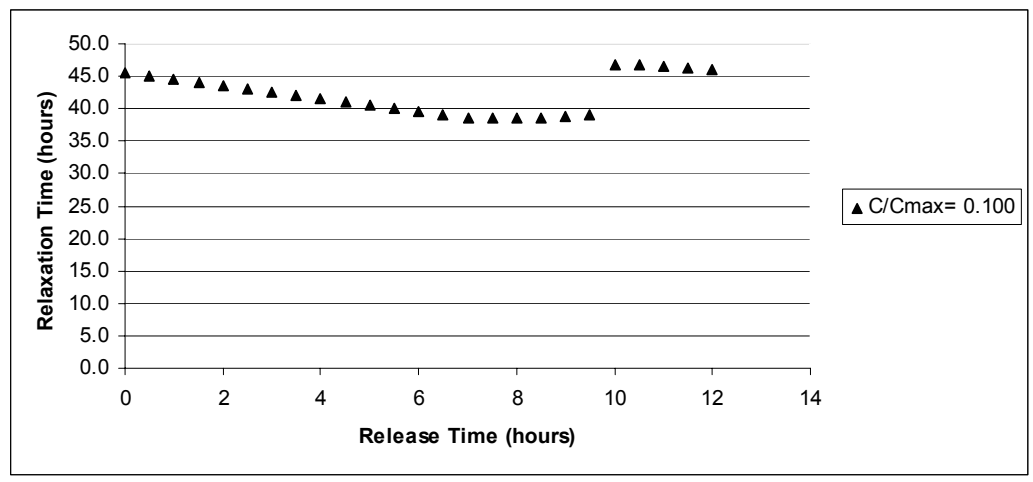

Figure 7: Time for a pollutant to reach $10 \%$ of its maximum value as a function of when the pollutant is released.

As an illustration, we consider an embayment with the same geometry used in the previous sections of this paper. We assume that a given mass of pollutant must be released and will be done so instantaneously (over a time equal to one step in the numerical integration of the governing mass flow equations in Stella). Further, it is assumed that it is critical for the pollutant to be flushed to $10 \%$ of its maximum value in as short a period as possible. Figure 7 presents the results of the analysis required to decide when the pollutant should be released. For these conditions, the minimum relaxation time occurs for a release time of about 8 hours or a little less than two hours past low tide. While the relaxation time is relatively constant for release times an hour before and after the optimum, it should be noted that, if the release was made at the 10 hour mark (or about 4 hours past low tide) the relaxation time would increase substantially.

\section{Conclusions}

The MSB model, which has been used to model well-mixed basins experiencing a constant pollutant loading, can be extended to include alternative pollution 
loading functions. Basic pulse, ramp and user-defined functions representing real pollution release scenarios can be used in the model. Some of the systematics relating the concentration relaxation times as a function of pollutant release times for a square pulse function have been studied for alternative definitions of relaxation times noted by the fraction, $C / C_{\max }$, which is the ratio of the desired concentration to the maximum concentration in the basin. This type of analysis has been applied to answer questions such as: when should a pollutant be released in order to minimize the time for the resulting concentration to relax to a particular fraction of the maximum concentration that is experienced? Answers to such questions depend on a number of factors including the geometry and bathymetry of the basin, and the pollution return factor, $b$. There are many additional considerations to the question of optimal pollution loading, including the use of alternative release strategies. In addition, the prevailing tidal regime in the basin will affect the optimum release strategy. Hopefully, it is clear from this study that such features can be introduced readily into the MSB model and some of this work is currently under way for particular embayments in the United States.

\section{References}

[1] Mecca, S.J., Severino, C. \& Barber, R.W., Pollution flushing models in Stella, Development and Application of Computer Techniques to Environmental Studies X, eds. G. Latini, G. Passerini and C.A. Brebbia, pp. 125-134, WIT Press: Southampton, UK, 2004.

[2] Barber, R.W., Advances in Coastal Modeling, edited by V.C. Lakhan, Chapter 17, Analytical Modeling of Pollution Flushing in Well-Mixed Tidal Embayments, Elsevier Science B.V., 2003.

[3] Mecca, S.J., Severino, C. \& Barber, R.W., Pollution flushing in cascading tidal basins, WIT Transactions of the Built Environment, 78, eds. M. Da Conceicao Cunha and C.A. Brebbia, WIT Press: Southampton, UK, 2005.

[4] Sanford, L.P., Boicourt, W.C. \& Rives, S.R., Model for estimating tidal flushing of small embayments, Journal of Waterway, Port, Coastal and Ocean Engineering, 118 (6), pp. 635-654, 1992. 